\title{
ENERGY-BASED YIELD CRITERIA FOR ORTHOTROPIC MATERIALS, EXHIBITING STRENGTH-DIFFERENTIAL EFFECT. SPECIFICATION FOR SHEETS UNDER PLANE STRESS STATE
}

\begin{abstract}
A general proposition of an energy-based limit condition for anisotropic materials exhibiting strength-differential effect (SDE) based on spectral decomposition of elasticity tensors and the use of scaling pressure-dependent functions is specified for the case of orthotropic materials. A detailed algorithm (based on classical solutions of cubic equations) for the determination of elastic eigenstates and eigenvalues of the orthotropic stiffness tensor is presented. A yield condition is formulated for both two-dimensional and three-dimensional cases. Explicit formulas based on simple strength tests are derived for parameters of criterion in the plane case. The application of both criteria for the description of yielding and plastic deformation of metal sheets is discussed in detail. The plane case criterion is verified with experimental results from the literature.
\end{abstract}

Keywords: anisotropy, yield condition, plasticity, metal sheets

\section{Motivation}

A large number of various yield criteria and plastic potentials for anisotropic materials have already been developed since the beginning of the $20^{\text {th }}$ century. It is enough to mention only some of the most important: Mises [19], Burzyński [7], Hill [9-13], Logan and Hosford [18], Hoffman [14], Tsai-Wu [29], Karafillis-Boyce [15], Rychlewski [22], Barlat et al. [1-5,8]. A great number and variety of different criteria make it questionable whether further propositions are really needed, especially when none of the previous ones has been definitely confirmed or rejected for a considerably broad scope of use. However, most of the proposed criteria are merely mathematical approximations or interpolations using linear transformations leading to the simplest polynomials or power-laws, lacking any physical motivation or even interpretation. Contrary to such an approach, a new proposition was developed [20,27-28] which is based on elastic energy density decomposition for linear elastic anisotropic solids - introduced first by Rychlewski [21] - modified by the use of certain stress-state-dependent weighting functions, the idea suggested independently by Burzyński [7], Schleicher [23] and Mises [19].

Among all branches of industry, it is the plastic forming of metal sheets where a description of yielding and plastic deformation of anisotropic materials (rolled sheets) is most commonly and most extensively used. For this reason, the following considerations should be narrowed to the case of orthotropic sheets in the plane stress state. The paper is, above all, of theoretical character. Its aim is to detail the formulation of a general proposi- tion mentioned above and to derive explicit formulas for yield stresses and Lankford coefficients (R-values) in chosen load cases (uniaxial stress, pure shear, equibiaxial stress). It should be done for two possible formulations incorporating three- and two-dimensional stiffness tensor respectively. Obtained formulas enable direct validation of this proposition with experimental results.

\section{Energy density decomposition for orthotropic materials}

Although a plane stress-state is assumed, the strain state remains three-dimensional. For this reason, we shall at first consider a general, three-dimensional stress and strain state in a linear elastic orthotropic material (with three mutually orthogonal two-fold symmetry axes). In a Cartesian coordinate system, axes of which coincide with the material's symmetry axes, its stiffness tensor has the following form:

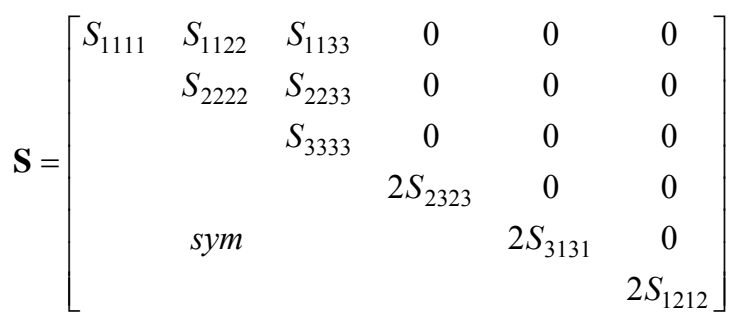

Spectral decomposition of elasticity tensors [21] for an orthotropic linear elastic material leads to a decomposition of elastic strain energy density as follows:

\footnotetext{
* CRACOW UNIVERSITY OF TECHNOLOGY, FACULTY OF CIVIL ENGINEERING, INSTITUTE OF STRUCTURAL MECHANICS, CRACOW, POLAND

\# Corresponding author: pszeptynski@pk.edu.pl
} 


$$
\Phi=\sum_{i=1}^{6} \Phi_{i} \quad \text { where } \quad \Phi_{i}=\frac{\left|\boldsymbol{\sigma}_{i}\right|^{2}}{2 \lambda_{i}}
$$

where $\lambda_{i}$ are eigenvalues of the stiffness tensor (Kelvin modulus) and $\left|\boldsymbol{\sigma}_{i}\right|$ are norms of the respective eigentensors. Such an additive decomposition (which is not an obvious thing for quadratic functions) is unique for given elasticity tensors. The weighted combination of components of this decomposition will be considered as a limit state criterion [20].

\subsection{Kelvin moduli and corresponding eigentensors}

The general form of spectral decomposition of orthotropic solids - namely methods of determination of Kelvin moduli and corresponding eigenstates - was discussed in $[6,17]$. In this section, I would like to propose an alternative complete algorithm for finding those quantities. Three of those energy density components correspond to pure shears in planes perpendicular to each of the symmetry axes, in the direction of the remaining two axes. The respective Kelvin moduli are simply the shear moduli in proper planes:

$$
\lambda_{4}=2 S_{2323} \quad \lambda_{5}=2 S_{3131} \quad \lambda_{6}=2 S_{1212}
$$

where $\mathrm{S}_{i j k l}$ are components of the stiffness tensor in a coordinate system, the axes of which coincide with three perpendicular symmetry axes of the material (main orthotropy axes). In all further considerations, it is assumed that the axes of the coordinate system coincide with the main ortothropy axes. The other three components correspond to some stress states with, in general, non-zero hydrostatic component. Either none of them is a purely hydrostatic stress or one of them is a hydrostatic stress state and the other two are some deviatoric states (at most, one of them may be a pure shear) orthogonal to those three mentioned above. As each of those three stress states has zero off-diagonal (shear) components, hence the main orthotropy axes are principal axes of each one of them. Kelvin moduli may be found by employing the standard procedure for finding the roots of a cubic equation:

1. We introduce:

$$
\begin{aligned}
& \bar{\lambda}=\frac{S_{1111}+S_{2222}+S_{3333}}{3} \\
& \mathbf{T}=\left[\begin{array}{lll}
S_{1111} & S_{1122} & S_{1133} \\
& S_{2222} & S_{2233} \\
\text { sym } & & S_{3333}
\end{array}\right] \\
& \mathbf{D}_{\mathbf{T}}=\left[\begin{array}{ccc}
S_{1111}-\bar{\lambda} & S_{1122} & S_{1133} \\
& S_{2222}-\bar{\lambda} & S_{2233} \\
\text { sym } & & S_{3333}-\bar{\lambda}
\end{array}\right] \\
& K_{2}=\frac{1}{2} \operatorname{tr}\left(\mathbf{D}_{\mathbf{T}} \cdot \mathbf{D}_{\mathbf{T}}\right) \quad K_{3}=\operatorname{det} \mathbf{D}_{\mathbf{T}} \\
& \Delta=\left(\frac{K_{3}}{2}\right)^{2}-\left(\frac{K_{2}}{3}\right)^{3}
\end{aligned}
$$

2. Then we may consider three cases:

a) $\Delta=0, K_{3}=0 \rightarrow$ three equal eigenvalues (such a situation occurs only when all Poisson's ratios are equal to 0 ): $\lambda_{1}=\lambda_{2}=\lambda_{3}=\lambda$

b) $\Delta=0, K_{3} \neq 0 \rightarrow$ one single and one double eigenvalue:

$$
\lambda_{1}=\lambda_{2}=\bar{\lambda}-\sqrt[3]{\frac{K_{3}}{2}} \quad \lambda_{3}=\bar{\lambda}+2 \sqrt[3]{\frac{K_{3}}{2}}
$$

c) $\Delta \neq 0, K_{3} \neq 0 \rightarrow$ three distinct eigenvalues.

$$
\lambda_{i}=\bar{\lambda}+\sqrt{\frac{4}{3} K_{2}} \cos \left[\begin{array}{l}
\frac{1}{3} \arccos \left(\frac{3 \sqrt{3}}{2} \frac{K_{3}}{K_{2}^{3 / 2}}\right)+ \\
+(i-1) \frac{2 \pi}{3}
\end{array}\right] i=1,2,3
$$

Projections of the stress state on eigensubspaces corresponding with the Kelvin moduli introduced above are:

$$
\left[\begin{array}{l}
\sigma_{1} \\
\sigma_{2} \\
\sigma_{3} \\
\sigma_{4} \\
\sigma_{5} \\
\sigma_{6}
\end{array}\right]=\left[\begin{array}{llllll}
p_{11} & p_{12} & p_{13} & & & \\
p_{21} & p_{22} & p_{23} & & & \\
p_{31} & p_{32} & p_{33} & & & \\
& & & 1 & & \\
& & & & 1 & \\
& & & & & 1
\end{array}\right] \cdot\left[\begin{array}{l}
\sigma_{11} \\
\sigma_{22} \\
\sigma_{33} \\
\sigma_{23} \\
\sigma_{31} \\
\sigma_{12}
\end{array}\right]
$$

where

$$
\begin{aligned}
& p_{11}=\cos \kappa_{1} \cos \kappa_{2} \\
& p_{12}=\cos \kappa_{1} \sin \kappa_{2} \\
& p_{13}=\sin \kappa_{1} \\
& p_{21}=-\cos \kappa_{3} \sin \kappa_{2}+\sin \kappa_{3} \sin \kappa_{1} \cos { } \\
& p_{22}=\cos \kappa_{3} \cos \kappa_{2}+\sin \kappa_{3} \sin \kappa_{1} \sin \kappa_{2} \\
& p_{23}=-\sin \kappa_{3} \cos \kappa_{1} \\
& p_{31}=\sin \kappa_{3} \sin \kappa_{2}+\cos \kappa_{3} \sin \kappa_{1} \cos \kappa_{2} \\
& p_{32}=-\sin \kappa_{3} \cos \kappa_{2}+\cos \kappa_{3} \sin \kappa_{1} \sin \kappa_{2} \\
& p_{33}=-\cos \kappa_{3} \cos \kappa_{1}
\end{aligned}
$$

and parameters $\kappa_{1}, \kappa_{2}, \kappa_{3}$ are closely related with non-dimensional invariants of the stiffness tensor termed stiffness distributors. Parameters $p_{i j}$ can be found as normalized solution of a linear system of dependent equations:

$$
\left[\begin{array}{ccc}
\left(S_{1111}-\lambda_{i}\right) & S_{1122} & S_{1133} \\
S_{1122} & \left(S_{2222}-\lambda_{i}\right) & S_{2233} \\
S_{1133} & S_{2233} & \left(S_{3333}-\lambda_{i}\right)
\end{array}\right] \cdot\left[\begin{array}{c}
p_{i 1} \\
p_{i 2} \\
p_{i 3}
\end{array}\right]=\left[\begin{array}{l}
0 \\
0 \\
0
\end{array}\right]
$$

Employing the standard procedure for finding eigenvectors of a $3 \times 3$ matrix, parameters $p_{i j}$ can be found as components of a normalized cross product of any two independent rows of the matrix:

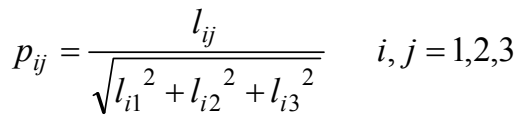

where

$$
\left\{\begin{array}{l}
l_{i 1}=\left(T_{22}-\lambda_{i}\right)\left(T_{33}-\lambda_{i}\right)-T_{23}{ }^{2} \\
l_{i 2}=T_{31} T_{23}-T_{12}\left(T_{33}-\lambda_{i}\right) \\
l_{i 3}=T_{12} T_{23}-T_{31}\left(T_{22}-\lambda_{i}\right)
\end{array}\right.
$$


or

$$
\left\{\begin{array}{l}
l_{i 1}=T_{23} T_{31}-T_{12}\left(T_{33}-\lambda_{i}\right) \\
l_{i 2}=\left(T_{11}-\lambda_{i}\right)\left(T_{33}-\lambda_{i}\right)-T_{13}{ }^{2} \\
l_{i 3}=T_{12} T_{31}-T_{23}\left(T_{11}-\lambda_{i}\right)
\end{array}\right.
$$

or

$$
\left\{\begin{array}{l}
l_{i 1}=T_{23} T_{12}-T_{31}\left(T_{22}-\lambda_{i}\right) \\
l_{i 2}=T_{31} T_{12}-T_{23}\left(T_{11}-\lambda_{i}\right) \\
l_{i 3}=\left(T_{11}-\lambda_{i}\right)\left(T_{22}-\lambda_{i}\right)-T_{12}{ }^{2}
\end{array}\right.
$$

and $T_{i j}$ are components of matrix $\mathbf{T}$ introduced in (2.4). In the case of a single eigenvalue, at least two of the above formulas provide a non-zero solution. In the case of one single and one double eigenvalue, the eigenstate corresponding with the single eigenvalue may be determined in a similar way, while two others may be any states with zero off-diagonal components, both of them being orthogonal to the former one. The case of a triple eigenvalue is a trivial one since any state with zero off-diagonal components is an eigenstate and any three such states constitute a basis.

Parameters $\kappa_{1}, \kappa_{2}, \kappa_{3}$ can be found as:

$$
\kappa_{1}=\arcsin p_{13} \quad \kappa_{2}=\operatorname{arctg} \frac{p_{12}}{p_{11}} \quad \kappa_{3}=\operatorname{arctg} \frac{p_{23}}{p_{33}}
$$

The algorithm presented in the paper provides not a unique projection of the current stress state on a proper eigensubspace but only a single normalized eigenstate $\mathbf{p}_{\mathbf{i}}$ which may be arbitrarily taken with either "+" or "_" sign. The choice of one of those solutions depends on what we choose to call a "positive" or "negative" eigenstate. As in the case of tension / compression, it is a matter of convention that we consider tension positive (e.g. hydrostatic pressure is very often signed in an opposite way). Nevertheless, the projection (which is unique, sign-independent) is calculated as $\boldsymbol{\sigma}_{\mathbf{i}}=\left(\boldsymbol{\sigma} \cdot \mathbf{p}_{\mathbf{i}}\right) \mathbf{p}_{\mathbf{i}}$ in which the sign of $\mathbf{p}_{\mathbf{i}}$ plays no role.

\section{Yield condition}

We may consider the following limit state condition:

$$
\sum_{i=1}^{6} \eta_{i}\left(\sigma_{i}\right) \Phi_{i}=1
$$

where $\Phi_{i}$ are components of the main orthogonal decomposition of elastic energy density for orthotropic material, $\sigma_{i}$ are projections of the stress state on the corresponding eigensubspace of stiffness tensor and $\eta_{i}$ are weighting functions termed influence functions. As is indicated by experiments, yield surface is often well described by a proper quadric surface which provides a description of the strength-differential effect [24-26] in uniaxial states. According to (2.2), each energy component $\Phi_{i}$ is proportional to $\left|\boldsymbol{\sigma}_{\mathbf{i}}\right|^{2}$. For this reason, we may assume the influence functions for states $\boldsymbol{\sigma}_{\mathbf{i}}(i=1,2,3)$ - incorporating pressure-dependent state or states which are always present in uniaxial states - as rational functions of the type proposed by Burzyński [7]:

$$
\eta_{i}\left(\sigma_{i}\right)=A_{i}+\frac{B_{i}}{\sigma_{i}} \quad(i=1,2,3)
$$

Assuming that the material is also orthotropic in the plastic regime, the strength-differential effect is not observed for pure shears $\boldsymbol{\sigma}_{\mathbf{i}}(i=4,5,6)$ and thus the yield condition is assumed to be in the form:

$$
f=\sum_{i=1}^{6} A_{i} \sigma_{i}^{2}+\sum_{j=1}^{3} B_{j} \sigma_{j}-1=0
$$

The condition depends now on, in general, 12 independent parameters: nine constant parameters $A_{i}, B_{j}$ and three independent functions of stiffness distributors $\kappa_{1}, \kappa_{2}, \kappa_{3}$ from which one can calculate nine parameters $p_{i j}$ determining the mode of eigentensors $\boldsymbol{\sigma}_{\mathbf{i}}(i=1,2,3)$ of the stiffness tensor. For materials exhibiting no strength-differential effect, it seems reasonable to take $B_{1}=B_{2}=B_{3}=0$.

Let us consider any stress state in which all its components are proportional to a single measure of stress $\sigma$ (e.g. uniaxial tension, uniaxial compression, equibiaxial tension, shear stress), namely stress states of form $\boldsymbol{\sigma}=\sigma \mathbf{N}$, where $\mathbf{N}$ is a certain normalized state of the assumed form. The choice between two different states $\mathbf{N}$ and $\mathbf{- N}$ is a matter of arbitrary decision, e.g. when measures $\sigma$ of both uniaxial tension and compression are meant to be positive, then $\mathbf{N}=\mathbf{n} \otimes \mathbf{n}$ should be taken in the former case, and $\mathbf{- N}$ in the latter. The stress state projections on eigenstates may be written as:

$$
\sigma_{i}=\sigma p_{i} \quad i=1,2, \ldots, 6
$$

where $p_{i}=\mathbf{P}_{i} \cdot \mathbf{N}\left(\mathbf{P}_{i}\right.$ is an orthogonal projector on the $i$-th eigensubspace) is the projection of normalized stress state on a proper eigensubspace. The yield condition takes the form of a quadratic equation with respect to $\sigma$ :

$$
\begin{aligned}
& A \sigma^{2}+B \sigma-1=0 \\
& \text { where } A=\sum_{i=1}^{6} A_{i} p_{i}^{2} \quad B=\sum_{j=1}^{3} B_{j} p_{j}
\end{aligned}
$$

The limit value of the stress measure $\sigma$, at which material yields, is equal:

$$
\sigma_{0}=\frac{-B \pm \sqrt{B^{2}+4 A}}{2 A}
$$

The equation will have, in general, two distinct roots. In the case of uniaxial load, they correspond with tensile and compressive strength. We will now consider a stress state in plane $\left(x_{1}, x_{2}\right)$ :

$$
\boldsymbol{\sigma}=\left[\begin{array}{ll}
\sigma_{11} & \sigma_{12} \\
\sigma_{12} & \sigma_{22}
\end{array}\right]
$$

In such a case, the projection on the fourth and fifth eigensubspace is zero and so it will be for any plane stress state in plane $\left(x_{1}, x_{2}\right)$. This is because, by taking $A_{4}=A_{5}=0$, we may cancel terms corresponding with those eigenstates in the yield criterion without any loss of generality. 


\subsection{Uniaxial stress state (tension / compression)}

Let us assume that the first axis of the coordinate system coincides with the rolling direction. Uniaxial stress state of magnitude $\sigma$ oriented at angle $\phi$ to the rolling direction (measured counterclockwise) is described as follows:

$$
\boldsymbol{\sigma}=\left[\begin{array}{ll}
\sigma_{11} & \sigma_{12} \\
\sigma_{12} & \sigma_{22}
\end{array}\right]=\sigma\left[\begin{array}{cc}
\cos ^{2} \phi & \cos \phi \sin \phi \\
\cos \phi \sin \phi & \sin ^{2} \phi
\end{array}\right]
$$

We may now project this state on appropriate eigenstates:

$$
\begin{aligned}
& \sigma_{1 u}(\phi)=\sigma\left(p_{11} \cos ^{2} \phi+p_{12} \sin ^{2} \phi\right)=\sigma p_{1 u}(\phi) \\
& \sigma_{2 u}(\phi)=\sigma\left(p_{21} \cos ^{2} \phi+p_{22} \sin ^{2} \phi\right)=\sigma p_{2 u}(\phi) \\
& \sigma_{3 u}(\phi)=\sigma\left(p_{31} \cos ^{2} \phi+p_{32} \sin ^{2} \phi\right)=\sigma p_{3 u}(\phi) \\
& \sigma_{6 u}(\phi)=\sigma(\cos \phi \sin \phi)=\sigma p_{6 u}(\phi)
\end{aligned}
$$

and $\sigma_{4 \mathrm{u}}=\sigma_{5 \mathrm{u}}=0$. Denoting:

$$
\begin{aligned}
& A_{u}(\phi)=A_{1} p_{1 u}^{2}+A_{2} p_{2 u}^{2}+A_{3} p_{3 u}^{2}+A_{6} p_{6 u}^{2} \\
& B_{u}(\phi)=B_{1} p_{1 u}+B_{2} p_{2 u}+B_{3} p_{3 u}
\end{aligned}
$$

uniaxial strength when loading at angle $\phi$ to the rolling direction may be expressed as:

$$
k_{u}(\phi)=\frac{-B_{u}(\phi) \pm \sqrt{B_{u}^{2}(\phi)+4 A_{u}(\phi)}}{2 A_{u}(\phi)}
$$

The two roots of the quadratic equation provided by the yield condition written for uniaxial stress state correspond with tensile and compressive strength. Of course, in the case of material exhibiting no strength-differential effect, proper modelling would require $B_{u}(\phi)=0$ and $k_{u}(\phi)= \pm A_{u}^{-1 / 2}(\phi)$.

\subsection{Pure shear}

Pure shear stress state in plane $\left(x_{1}, x_{2}\right)$, with shear stresses $\tau$ oriented at angle $\phi$ (measured counterclockwise) to the rolling direction is described by tensor:

$$
\boldsymbol{\sigma}=\left[\begin{array}{ll}
\sigma_{11} & \sigma_{12} \\
\sigma_{12} & \sigma_{22}
\end{array}\right]=\tau\left[\begin{array}{cc}
-\sin 2 \phi & \cos 2 \phi \\
\cos 2 \phi & \sin 2 \phi
\end{array}\right]
$$

We may now project this state on appropriate eigenstates:

$$
\begin{aligned}
& \sigma_{1 s}(\phi)=\tau\left[\left(p_{12}-p_{11}\right) \sin 2 \phi\right]=\tau p_{1 s}(\phi) \\
& \sigma_{2 s}(\phi)=\tau\left[\left(p_{22}-p_{21}\right) \sin 2 \phi\right]=\tau p_{2 s}(\phi) \\
& \sigma_{3 s}(\phi)=\tau\left[\left(p_{32}-p_{31}\right) \sin 2 \phi\right]=\tau p_{3 s}(\phi) \\
& \sigma_{6 s}(\phi)=\tau[\cos 2 \phi]=\tau p_{6 s}(\phi)
\end{aligned}
$$

$\sigma_{4 s}=\sigma_{5 s}=0$. Limit stress is expressed in an analogous way as in previous cases.

\subsection{Equibiaxial stress state}

Equibiaxial stress state of magnitude $\sigma$ oriented at any angle to the rolling direction is described as follows:

$$
\boldsymbol{\sigma}=\left[\begin{array}{ll}
\sigma_{11} & \sigma_{12} \\
\sigma_{12} & \sigma_{22}
\end{array}\right]=\sigma\left[\begin{array}{ll}
1 & 0 \\
0 & 1
\end{array}\right]
$$

We may now project this state on appropriate eigenstates:

$$
\begin{aligned}
& \sigma_{1 b}=\sigma\left(p_{11}+p_{12}\right)=\sigma p_{1 b} \\
& \sigma_{2 b}=\sigma\left(p_{21}+p_{22}\right)=\sigma p_{2 b} \\
& \sigma_{3 b}=\sigma\left(p_{31}+p_{32}\right)=\sigma p_{3 b}
\end{aligned}
$$

$\sigma_{4 b}=\sigma_{5 b}=\sigma_{6 b}=0$. Limit stress is expressed in an analogous way as in previous cases.

\section{Plastic deformation}

One may use the yield condition function as a plastic potential

$$
\psi(\boldsymbol{\sigma})=\sum_{i=1}^{6} A_{i} \sigma_{i}^{2}+\sum_{j=1}^{3} B_{j} \sigma_{j}
$$

in an associated flow rule of Levy-Mises equations:

$$
\mathrm{d} \varepsilon_{i j}^{p}=\mathrm{d} \Lambda \frac{\partial \psi}{\partial \sigma_{i j}}
$$

Although yield condition (3.3) may be well interpreted in terms of elastic energy density, it is no longer so in the case of plastic potential (4.1) - since the stress-strain relation becomes non-linear after yielding, elastic energy density cannot be any longer decomposed into (2.2). However, function (4.1) may be still a fair approximation of the plastic potential as it is indicated by broad use of Levy-Mises equations with a flow rule associated with Maxwell-Huber-Mises-Hencky yield criterion for isotropic solids, which has clear energetic interpretation within the elastic range. Dependency of plastic potential on elastic constants (via parameters $\kappa_{\mathrm{i}}$ ) may raise further questions, due to permanent changes in the internal structure of the material and due to progressive induction of anisotropy during plastic deformation. It is known, however, that elastic constants themselves remain almost unchanged during plastic deformation so function (4.1) may still provide a valuable proposition for a plastic potential even if it lacks clear interpretation after reaching the yield point.

Components of the plastic strain rate tensor may be now calculated. We will be interested in the strain rate components which enable us to calculate Lankford coefficients (plastic strain ratios, R-values):

$$
\begin{aligned}
& \mathrm{R}_{\phi}^{\mathrm{u}}=\frac{\mathrm{d} \varepsilon_{2^{\prime} 2^{\prime}}^{p}}{\mathrm{~d} \varepsilon_{3^{\prime} 3^{\prime}}^{p}}-\mathrm{R} \text {-value for uniaxial load } \\
& \mathrm{R}_{\phi}^{\mathrm{b}}=\frac{\mathrm{d} \varepsilon_{2^{\prime} 2^{\prime}}^{p}}{\mathrm{~d} \varepsilon_{1^{\prime} 1^{\prime}}^{p}}-\mathrm{R} \text {-value for equibiaxial load } \\
& \mathrm{R}_{\phi}^{\mathrm{s}}=\frac{\mathrm{d} \varepsilon_{1^{\prime} 2^{\prime}}^{p}}{\mathrm{~d} \varepsilon_{3^{\prime} 3^{\prime}}^{p}}-\mathrm{R} \text {-value for pure shear load }
\end{aligned}
$$


In the above formulas, direction 1' denotes the load direction (which, in general, does not coincide with the rolling direction denoted with 1), direction $2^{\prime}$ is the one in the plane of the sheet and perpendicular to the load direction and direction 3 ' is the through-thickness direction. Components of the strain increment tensor in the coordinate system in which the first axis is parallel to the rolling direction are obtained through differentiation of the plastic potential:

$$
\begin{aligned}
& \mathrm{d} \varepsilon_{11}^{p}=\mathrm{d} \Lambda \frac{\partial \psi}{\partial \sigma_{11}}=\mathrm{d} \Lambda\left[\sum_{i=1}^{3}\left(2 A_{i} \sigma_{i} p_{i 1}+B_{i} p_{i 1}\right)\right] \\
& \mathrm{d} \varepsilon_{22}^{p}=\mathrm{d} \Lambda \frac{\partial \psi}{\partial \sigma_{22}}=\mathrm{d} \Lambda\left[\sum_{i=1}^{3}\left(2 A_{i} \sigma_{i} p_{i 2}+B_{i} p_{i 2}\right)\right] \\
& \mathrm{d} \varepsilon_{33}^{p}=\mathrm{d} \Lambda \frac{\partial \psi}{\partial \sigma_{33}}=\mathrm{d} \Lambda\left[\sum_{i=1}^{3}\left(2 A_{i} \sigma_{i} p_{i 3}+B_{i} p_{i 3}\right)\right] \\
& \mathrm{d} \varepsilon_{12}^{p}=\mathrm{d} \Lambda \frac{\partial \psi}{\partial \sigma_{12}}=\mathrm{d} \Lambda\left[2 A_{6} \sigma_{6}\right]
\end{aligned}
$$

Values corresponding to uniaxial load at angle $\phi$ to the rolling direction are obtained by substitution of $\sigma_{i u}$ defined in section 3.1 instead of $\sigma_{i}$-similarly, for pure shear and equibiaxial load, we shall use $\sigma_{i s}$ (section 3.2) and $\sigma_{i b}$ (section 3.3) respectively. Having determined the plastic strain increment tensor, we may calculate components needed to calculate the Lankford coefficients using transformation formulas:

$$
\begin{aligned}
& \mathrm{d} \varepsilon_{1^{\prime} 1^{\prime}}^{p}=\mathrm{d} \varepsilon_{11}^{p} \cos ^{2} \phi+\mathrm{d} \varepsilon_{22}^{p} \sin ^{2} \phi+\mathrm{d} \varepsilon_{12}^{p} \sin 2 \phi \\
& \mathrm{d} \varepsilon_{2^{\prime} 2^{\prime}}^{p}=\mathrm{d} \varepsilon_{11}^{p} \sin ^{2} \phi+\mathrm{d} \varepsilon_{22}^{p} \cos ^{2} \phi-\mathrm{d} \varepsilon_{12}^{p} \sin 2 \phi \\
& \mathrm{d} \varepsilon_{1^{\prime} 2^{\prime}}^{p}=\frac{1}{2}\left(\mathrm{~d} \varepsilon_{22}^{p}-\mathrm{d} \varepsilon_{11}^{p}\right) \sin 2 \phi+\mathrm{d} \varepsilon_{12}^{p} \cos 2 \phi \\
& \mathrm{d} \varepsilon_{3^{\prime} 3^{\prime}}^{p}=\mathrm{d} \varepsilon_{33}^{p}
\end{aligned}
$$

Please note, that, in general, the incompressibility condition $\mathrm{d} \varepsilon_{11}^{p}+\mathrm{d} \varepsilon_{22}^{p}+\mathrm{d} \varepsilon_{33}^{p}=0$ may not be fulfilled, as it depends on elastic and strength properties of the material incorporated in parameters $p_{i j}$ and $A_{i}, B_{i}$ respectively. It is closely related to pressure-dependency of the yield criterion with which plastic potential is associated. If a hydrostatic state is an eigenstate of the elasticity tensors (so called "volumetrically isotropic materials") and the yield condition assumes that an influence function corresponding with this state is constantly equal zero, then both the yield function and the plastic potential associated with it are pressure-independent and describe plastically incompressible material.

\section{Alternative proposition of yield criterion based on spectral decomposition of the plane stiffness tensor}

All above considerations may be similarly done based on spectral decomposition of the plane stiffness tensor [27]:

$$
\mathbf{S}=\left[\begin{array}{ccc}
S_{1111} & S_{1122} & 0 \\
S_{1122} & S_{2222} & 0 \\
0 & 0 & 2 S_{1212}
\end{array}\right]
$$

Components of the stiffness tensor may be expressed in terms of the most commonly used measures of stiffness, namely the longitudinal stiffness modulus (Young modulus) and the transverse stiffness modulus (Kirchhoff modulus), i.e. as:

$$
\begin{aligned}
& S_{1111}=\frac{E_{1}^{2}}{E_{1}-E_{2} v_{12}^{2}}, \quad S_{2222}=\frac{E_{1} E_{2}}{E_{1}-E_{2} v_{12}^{2}}, \\
& S_{1122}=\frac{E_{1} E_{2} v_{12}}{E_{1}-E_{2} v_{12}^{2}}, \quad S_{1212}=G
\end{aligned}
$$

where $E_{1}$ and $E_{2}$ denotes the Young modulus along the rolling direction and along the transverse direction respectively, $v_{12}$ is the Poisson's ratio when stretching along the rolling direction and $G$ is the Kirchhoff modulus. Similar formulas may be written incorporating ratio $v_{21}$. In this case, the main decomposition of elastic energy density is as follows:

$$
\Phi=\sum_{i=1}^{3} \Phi_{i} \quad \text { where } \Phi_{i}=\frac{\sigma_{i}^{2}}{2 \lambda_{i}}
$$

and

$$
\left[\begin{array}{l}
\sigma_{1} \\
\sigma_{2} \\
\sigma_{3}
\end{array}\right]=\left[\begin{array}{ccc}
\cos \kappa & \sin \kappa & \\
-\sin \kappa & \cos \kappa & \\
& & 1
\end{array}\right] \cdot\left[\begin{array}{l}
\sigma_{11} \\
\sigma_{22} \\
\sigma_{12}
\end{array}\right]
$$

where the Kelvin moduli are equal

$$
\begin{aligned}
& \lambda_{1}=\frac{2 E_{1} E_{2}}{E_{2}+E_{1}-\sqrt{\left(E_{2}-E_{1}\right)^{2}+4 v_{12}^{2} E_{2}^{2}}} \\
& \lambda_{2}=\frac{2 E_{1} E_{2}}{E_{2}+E_{1}+\sqrt{\left(E_{2}-E_{1}\right)^{2}+4 v_{12}^{2} E_{2}^{2}}} \\
& \lambda_{3}=2 G
\end{aligned}
$$

and parameter $\kappa$ is a function of the stiffness distributor and it is given as

$$
\kappa=-\operatorname{arctg}\left[\frac { E _ { 1 } } { 2 v _ { 1 2 } } \left(\frac{1}{E_{2}}-\frac{1}{E_{1}}-\sqrt{\left.\left.\left(\frac{1}{E_{2}}-\frac{1}{E_{1}}\right)^{2}+\frac{4 v_{12}^{2}}{E_{1}^{2}}\right)\right]}\right.\right.
$$

The yield condition using the Burzyński-type influence function becomes:

$$
f=A_{1} \sigma_{1}^{2}+B_{1} \sigma_{1}+A_{2} \sigma_{2}^{2}+B_{2} \sigma_{2}+A_{3} \sigma_{3}^{2}-1=0
$$

Parameters $A_{i}(i=1,2,3) B_{j}(j=1,2)$ may be uniquely determined knowing the tensile and compressive strength of the material along the rolling direction and along the direction perpendicular to it $k_{t 1}, k_{t 2}, k_{c 1}, k_{c 2}$ and knowing the limit shear stress $k_{s}$ when shearing along those directions:

$$
\begin{aligned}
& A_{1}=\frac{1}{k_{t 1}^{2}}\left[\begin{array}{l}
\frac{\sin ^{2} \kappa}{K^{2} k_{2}\left(\sin ^{4} \kappa-\cos ^{4} \kappa\right)}+ \\
-\frac{\cos ^{2} \kappa}{k_{1}\left(\sin ^{4} \kappa-\cos ^{4} \kappa\right)}
\end{array}\right] \\
& B_{1}=\frac{1}{k_{t 1}}\left[\cos \kappa \frac{k_{1}-1}{k_{1}}+\frac{\sin \kappa}{K} \frac{k_{2}-1}{k_{2}}\right]
\end{aligned}
$$




$$
\begin{aligned}
& A_{2}=\frac{1}{k_{t 1}^{2}}\left[\begin{array}{l}
\frac{\sin ^{2} \kappa}{k_{1}\left(\sin ^{4} \kappa-\cos ^{4} \kappa\right)}+ \\
\left.-\frac{\cos ^{2} \kappa}{K^{2} k_{2}\left(\sin ^{4} \kappa-\cos ^{4} \kappa\right)}\right]
\end{array}\right. \\
& B_{2}=\frac{1}{k_{t 1}}\left[\frac{\cos \kappa}{K} \frac{k_{2}-1}{k_{2}}-\sin \kappa \frac{k_{1}-1}{k_{1}}\right] \\
& A_{3}=\frac{1}{k_{s}^{2}}
\end{aligned}
$$

where

$$
K=\frac{k_{t 2}}{k_{t 1}} \quad k_{1}=\frac{k_{c 1}}{k_{t 1}} \quad k_{2}=\frac{k_{c 2}}{k_{t 2}}
$$

Instead of these parameters, the whole criterion may be determined with a different set of strength quantities. Shear strength - often difficult to determine precisely for metal sheets - may be replaced, i.e. with a tensile or compressive strength along the direction aligned to the rolling direction at an angle, e.g. $45^{\circ}$ :

$$
\begin{aligned}
& k_{s}=\left[\sqrt{\frac{4}{k_{t 45}^{2}}-\frac{2}{k_{t 45}} B_{45}-A_{45}}\right]^{-1} \\
& k_{s}=\left[\sqrt{\frac{4}{k_{c 45}^{2}}+\frac{2}{k_{c 45}} B_{45}-A_{45}}\right]^{-1}
\end{aligned}
$$

where:

$$
\begin{aligned}
& A_{45}=A_{1}(\cos \kappa+\sin \kappa)^{2}+A_{2}(\cos \kappa-\sin \kappa)^{2} \\
& B_{45}=B_{1}(\cos \kappa+\sin \kappa)+B_{2}(\cos \kappa-\sin \kappa)
\end{aligned}
$$

All the above formulas simplify much if the material is considered symmetric. Namely, when tensile and compressive strength for each direction are considered equal: $k_{1}=k_{2}=1$. Having completely determined the criterion, we are able to find strength in an arbitrary chosen stress state, e.g. uniaxial state, pure shear or equibiaxial state. They can be found easily using formulas analogous to equations (3.10) and (3.11). Pure shear in the direction aligned at a given angle $\phi$ to the rolling direction is of greater interest. Let us consider the case of $\phi=45^{\circ}$. In the limit state, the stress state components are $\sigma_{11}=-\sigma_{22}=k_{s 45}$, the rest of them being zero. Denoting:

$$
\begin{aligned}
& A=A_{1}(\cos \kappa-\sin \kappa)^{2}+A_{2}(\cos \kappa+\sin \kappa)^{2} \\
& B=B_{1}(\cos \kappa-\sin \kappa)-B_{2}(\cos \kappa+\sin \kappa)
\end{aligned}
$$

the solution is:

$$
k_{s 45}^{(1)}=\frac{-B-\sqrt{B^{2}+4 A}}{2 A} \quad k_{s 45}^{(2)}=\frac{-B+\sqrt{B^{2}+4 A}}{2 A}
$$

Two distinct roots of this equation indicate that there occurs a strength-differential effect in shearing. Namely, shear strength depends not only on the orientation of shearing direction but also on the sign of stresses. At first, it may seem in contradiction with our expectations. However, we must note that shear tests of sheet specimens are relatively rare and are difficult to be performed in such a way that the obtained shear is truly a pure one. The second thing is that, most often, such tests are performed along the rolling direction or perpendicularly to it and, in those two particular directions, the considered effect - according to the above proposition - does not occur. The most important reason for not denying this result is an obvious observation that comes from the interpretation of shearing at an angle $45^{\circ}$ to the rolling direction. Depending on the sign of the stress, either the fibres along the rolling direction are stretched and the perpendicular ones compressed or in an opposite way. Since the tensile and compressive strengths are assumed different for a single direction and also different between any two distinct directions, it is natural that strength-differential effect should occur also in shearing. For the same reasons, SDE should not be present when shearing along the rolling direction and the transverse one. In both cases, no matter the sign of the stress, the same fibres at an angle $45^{\circ}$ to the rolling direction are stretched and compressed - compare Fig. 1 and Fig. 2. This issue may be the subject of further experimental investigation.
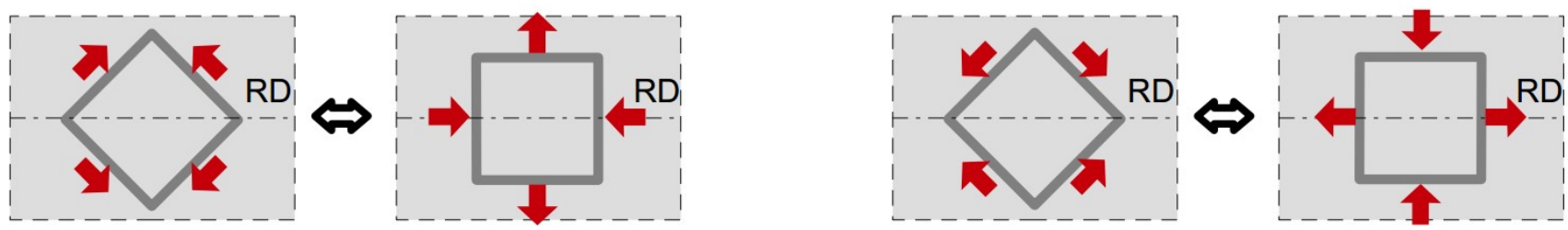

Fig. 1. Strength-differential effect (SDE) when shearing. Fibres along the rolling direction (RD) being stretched or compressed depending on the sign of the shear stress
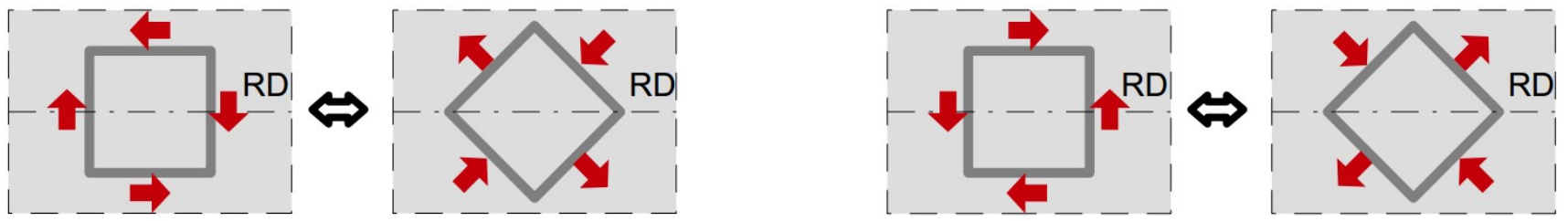

Fig. 2. Lack of SDE when shearing along the rolling direction (RD) and the transverse one 


\subsection{Experimental verification}

The proposed criterion was verified with the use of experimental data on Ti-6Al-4V titanium alloy given by Khan et al. [16].

TABLE 1

Tensile (t) and compressive (c) strength along the rolling direction and perpendicularly to it

\begin{tabular}{|c|c|c|c|c|}
\hline $\boldsymbol{k}_{\boldsymbol{t} \mathbf{1}}$ & $\boldsymbol{k}_{\boldsymbol{t} \mathbf{2}}$ & $\boldsymbol{k}_{\boldsymbol{c} \mathbf{1}}$ & $\boldsymbol{k}_{\boldsymbol{c} \mathbf{2}}$ & $\boldsymbol{k}_{\boldsymbol{s}}$ \\
\hline $955,71 \mathrm{MPa}$ & $902,55 \mathrm{MPa}$ & $1087,1 \mathrm{MPa}$ & $933,86 \mathrm{MPa}$ & $515,47 \mathrm{MPa}$ \\
\hline
\end{tabular}

TABLE 2

Compressive yield stress measured at different angles about the rolling direction

\begin{tabular}{|c|c|c|c|c|}
\hline $\boldsymbol{k}_{c}\left(\phi=\mathbf{0}^{\circ}\right)=\boldsymbol{k}_{c 1}$ & $\boldsymbol{k}_{c}\left(\phi=\mathbf{1 5}^{\circ}\right)$ & $\boldsymbol{k}_{c}\left(\phi=\mathbf{3 0}^{\circ}\right)$ & $\boldsymbol{k}_{c}\left(\phi=\mathbf{4 5}^{\circ}\right)$ & $\boldsymbol{k}_{c}\left(\phi=\mathbf{9 0}^{\circ}\right)$ \\
\hline
\end{tabular}

\begin{tabular}{|c|c|c|c|c|c|}
\hline $1087,1 \mathrm{MPa}$ & $1043,9 \mathrm{MPa}$ & $1021,2 \mathrm{MPa}$ & $992,95 \mathrm{MPa}$ & $933,86 \mathrm{MPa}$ \\
\hline
\end{tabular}

Due to the lack of data on the elastic properties of the considered titanium alloy, parameters $A_{i}(i=1,2,3) B_{j}(j=1,2$ were determined with the use of formulas (5.8)-(5.11), while parameter $\kappa$ was determined in such a way that the shear strength predicted by formula (5.7) fitted the one measured in the experiment. The following results were obtained:

$$
\begin{array}{ll}
\kappa=0,71254 & \\
A_{1}=3,0336 \cdot 10^{-7} & B_{1}=1,1998 \cdot 10^{-4} \\
A_{2}=1,8456 \cdot 10^{-6} & B_{2}=-5,5467 \cdot 10^{-5}
\end{array}
$$

The value of the function of the stiffness distributor $\kappa=0,71254$ for an average value of Poisson's ratio for Ti-6Al-4V alloy $v=0,342$ corresponds with the ratio of Young moduli along the rolling direction and along the transverse one $E_{1} / E_{2}=1,100$. Yield stresses along those two directions, both in tension and in compression, are predicted exactly, as well as limit shear stress and yield stress in compression at $45^{\circ}$. Using equation (5.7) with constants given by (5.14), one can calculate the predicted yield stress for different directions. The obtained results are presented in Figures 3 and 4 . The relative error of this estimation is 2,66\%.

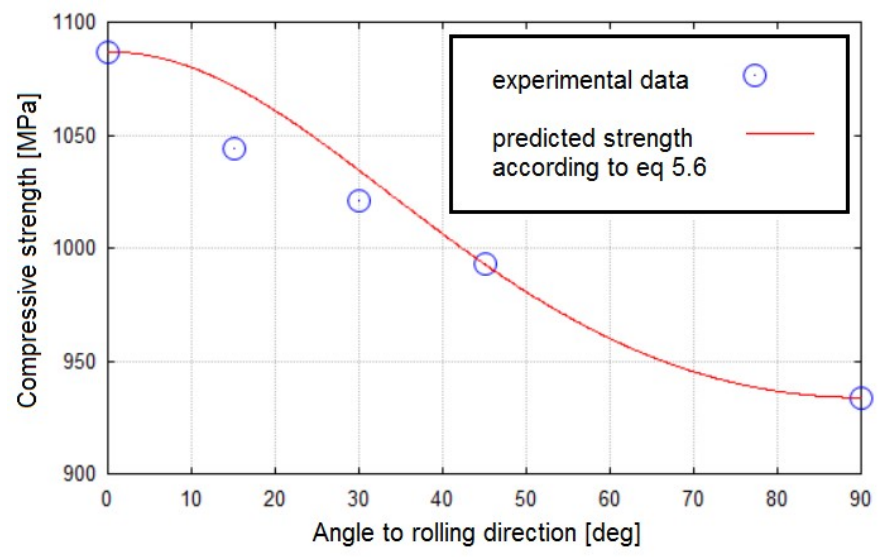

Fig. 3. Comparison of experimental results and compressive strength predicted by equation (5.7)

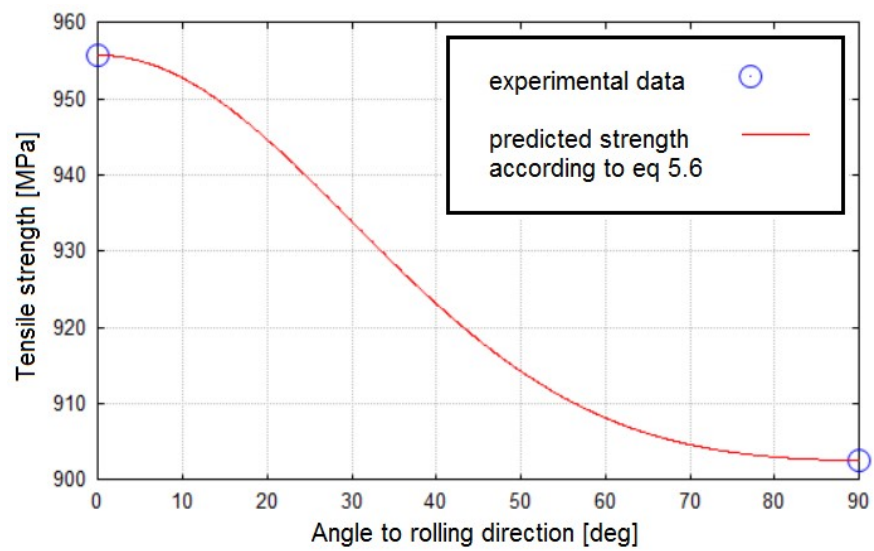

Fig. 4. Comparison of experimental results and tensile strength predicted by equation (5.7)

\subsection{Plastic deformation}

Based on the same considerations as in section 4.1, we may propose using an associated flow rule in Levy-Mises equations:

$$
\psi=A_{1} \sigma_{1}^{2}+B_{1} \sigma_{1}+A_{2} \sigma_{2}^{2}+B_{2} \sigma_{2}+A_{3} \sigma_{3}^{2}
$$

The components of the plastic strain increment tensor are equal:

$$
\begin{aligned}
& \mathrm{d} \varepsilon_{11}^{p}=\mathrm{d} \Lambda\left[\left(2 A_{1} \sigma_{1}+B_{1}\right) \cos \kappa-\left(2 A_{2} \sigma_{2}+B_{2}\right) \sin \kappa\right] \\
& \mathrm{d} \varepsilon_{22}^{p}=\mathrm{d} \Lambda\left[\left(2 A_{1} \sigma_{1}+B_{1}\right) \sin \kappa+\left(2 A_{2} \sigma_{2}+B_{2}\right) \cos \kappa\right] \\
& \mathrm{d} \varepsilon_{12}^{p}=\mathrm{d} \Lambda\left[2 A_{3} \sigma_{3}\right]
\end{aligned}
$$

Having described the problem with the plane stress tensor and the plane stiffness tensor, we cannot determine the plastic strain increment along the through-thickness direction, since the yield condition is independent of the corresponding stress. If we, however, narrow our consideration to plastically incompressible materials - which is the commonly accepted basis of the classical theory of plasticity - it can be found from the incompressibility condition:

$$
\mathrm{d} \varepsilon_{33}^{p}=-\left(\mathrm{d} \varepsilon_{11}^{p}+\mathrm{d} \varepsilon_{22}^{p}\right)
$$

Further calculation of Lankford coefficients are based on transformation formulas (4.6) and definitions given by equations (4.2)-(4.4).

\section{Summary}

Two propositions of a yield criterion providing a plastic potential for the description of plastic deformation of metal sheet with the use of an associated flow rule were presented. The first one - depending on 7 constant parameters - does not assume, in advance, incompressibility of material while the second one - depending on 5 constant parameters - is capable of determining the Lankford coefficients only when such an assumption is made. Closed algebraic formulas for strength at uniaxial stress, 
pure shear and equibiaxial stress states, as well as for plastic strain ratios in those states, were derived. They enable direct verification of the presented propositions with experimental data. Complete determination of the yield criterion also requires finding all the elastic constants - namely Young moduli and Poisson's ratios for three perpendicular directions in the case of the first proposition and for two directions in the case of the second one. The obtained criteria provide a quadratic yield surface and incorporate, in general, non-linear pressure dependency. They both have also an energetic interpretation in terms of the main decomposition of elastic energy density for anisotropic solids and assumed rational influence functions.

It must be, however, mentioned that if no energetic interpretation is needed, then both propositions provide us with a mathematical formulation which can be even more precise, as stiffness distributors' functions $\kappa_{i}(i=1,2,3)$ - which normally are determined by the elastic properties of a solid-may be then treated as additional fitting parameters. A valuable feature of this formulation is that stress state decomposition into parts $\sigma_{i}(i=1,2, \ldots, 6)$ remains an orthogonal decomposition. Further generalization may account also for non-associated flow rules of the same mathematical form but of different values of its parameters - its number increases up to 20 for the first proposition and 12 for the second one.

Simplified verification of criterion with experimental data, which was performed in this paper, indicates that it may be a useful tool for the estimation of the strength of anisotropic materials. However, more precise investigation is needed, accounting for measurement of both elastic and strength properties of the material. It may then become clear if stress state decomposition into elastic eigenstates provides a useful criterion of the yielding of anisotropic solids or if different decompositions or linear transformations of the actual stress state are better solutions.

\section{REFERENCES}

[1] F. Barlat, J. Lian, Int. J. Plast. 5, 51-61 (1989).

[2] F. Barlat, D.J. Lege, J.C. Brem, Int. J. Plast. 7, 693-712 (1991).

[3] F. Barlat, R.C. Becker, Y. Hayashida, Y. Maeda, M. Yanagawa, K. Chung, J.C. Brem, D.J. Lege, K. Matsui, S.J. Murtha, S. Hattori, Int. J. Plast. 13, 385-401 (1997).
[4] F. Barlat, Y. Maeda, K. Chung, M. Yanagawa, J.C. Brem, Y. Hayashida, D.J. Lege, K. Matsui, S.J. Murtha, S. Hattori, R.C. Becker, S. Makosey, J. Mech. Phys. Solids 45, 1727-1763 (1997).

[5] F. Barlat, J.C. Brem, J.W. Yoon, K. Chung, R.E. Dick, D.J. Lege, F. Pourboghrat, S.H. Choi, E. Chu, Int. J. Plasticity 19, 1297-1319 (2003).

[6] A. Blinowski, J. Ostrowska-Maciejewska, Arch. Mech. 48 (1), 129-141 (1996).

[7] W. Burzyński, Studium nad hipotezami wytężenia, $\mathrm{PhD}$ thesis, Akademia Nauk Technicznych, Lwów (1928).

[8] O. Cazacu, F. Barlat, Math. Mech. Solids 6 (6), 613-630 (2001).

[9] R. Hill, The mathematical theory of plasticity, 1950 Oxford University Press, Oxford.

[10] R. Hill, Math. Proc. Camb. Phil. Soc. 85, 179-190 (1979).

[11] R. Hill, J. Mech. Phys. Solids 38, 405-417 (1990).

[12] R. Hill, Int. J. Mech. Sci. 35 (1), 19-25 (1993).

[13] R. Hill, Proc. Roy. Soc. (London) A 193, 281-297 (1948).

[14] O. Hoffman, J. Comp. Mater. 1, 200-206 (1967).

[15] A.P. Karafillis, M.C. Boyce, J. Mech. Phys. Solids 12, 1859-1886 (1993).

[16] A.S. Khan, R. Kazmi, B. Farrokh, Int. J. Plast. 23, 931-950 (2007).

[17] K. Kowalczyk-Gajewska, J. Ostrowska-Maciejewska, Engng. Trans. 57 (3-4), 145-183 (2009).

[18] W.L. Logan, W.F. Hosford, Int. J. Mech. Sci. 22, 419-430 (1980).

[19] R. von Mises, ZAMM 8 (3), 161-185 (1928).

[20] J. Ostrowska-Maciejewska, P. Szeptyński, R.B. Pęcherski, Arch. Metall. Mater. 58 (4), 1223-1235 (2013).

[21] J. Rychlewski, J. Appl. Math. Mech. 48, 303-314 (1984).

[22] J. Rychlewski, For English translation see: Engng. Trans. 59 (1), 31-63 (2011).

[23] F. Schleicher, ZAMM 6, 199-216 (1926).

[24] W.A. Spitzig, R.J. Sober, O. Richmond, Acta Metall. 23, 885-894 (1975).

[25] W.A. Spitzig, R.J. Sober, O. Richmond, Metall. Trans. A 7A, 1703-1710 (1976).

[26] W.A. Spitzig, O. Richmond, Acta Metall. 32, 457-463 (1984).

[27] P. Szeptyński, R.B. Pęcherski, Rudy i Metale Nieżelazne 57 (4), 243-250 (2012).

[28] P. Szeptyński, Opracowanie kryterium stanu granicznego dla materiałów anizotropowych wykazujących asymetrię zakresu sprężystego, PhD thesis, AGH University of Science and Technology, Kraków, October 2013.

[29] S.W. Tsai, E.M. Wu, J. Comp. Mater. 5, 58-80 (1971). 